\title{
A NONLINEAR VOLTERRA EQUATION WITH RAPIDLY DECAYING SOLUTIONS \\ BY
}

OLOF J. STAFFANS

\begin{abstract}
We study the asymptotic behavior of the solutions of a nonlinear integrodifferential Volterra equation with a convolution kernel. More specifically, we give conditions which imply that a solution $x$ satisfies $x(t)=O\left(t^{-p}\right)(t \rightarrow \infty)$, where $p$ is an arbitrary, positive real number.
\end{abstract}

1. Introduction and summary of results. We study the asymptotic behavior of the solutions of the nonlinear integrodifferential equation

$$
x^{\prime}(t)+\int_{0}^{t} a(t-s) g(x(s)) d s=f(t) \quad\left(t \in R^{+}\right) .
$$

Here $R^{+}=[0, \infty), a, g$, and $f$ are given, real continuous functions, and $x$ is the unknown solution. In particular, we give conditions which imply that $x(t)=$ $O\left(t^{-p}\right)(t \rightarrow \infty)$ for $p>0$.

Our assumptions are the following (the notations are explained immediately after the list of assumptions; $p$ is a nonnegative real number).

$$
\begin{gathered}
a(t)=\alpha+a_{0}(t)\left(t \in R^{+}\right), \text {where } \alpha>0, \text { and } a_{0} \\
\text { is continuous and strongly positive definite on } R^{+}, \\
(1+t)^{p} a_{0}(t) \in L^{1}\left(R^{+}\right), \quad(1+t)^{p} a_{0}^{\prime}(t) \in L^{1} \cap \mathrm{BV}\left(R^{+}\right), \\
(1+t)^{p} f(t),(1+t)^{p} f^{\prime}(t) \in L^{2}\left(R^{+}\right), \\
g \in C(R), \begin{array}{r}
\xi g(\xi)>0 \quad(\xi \neq 0), \quad-\int_{-\infty}^{0} g(\xi) d \xi=\int_{0}^{\infty} g(\xi) d \xi=\infty, \\
\liminf _{\xi \rightarrow 0} g(\xi) / \xi>0, \quad \underset{\xi \rightarrow 0}{\lim \sup } g(\xi) / \xi<\infty .
\end{array}
\end{gathered}
$$

Here the strong positive definiteness of $a_{0}$ means that there should exist $\varepsilon>0$ such that $a_{0}(|t|)-\varepsilon e^{-|t|}(t \in R)$ is a (Bochner) positive definite function. The statements concerning $a^{\prime}$ and $f^{\prime}$ should be interpreted as requirements that $a, f$ be locally absolutely continuous, together with the given conditions on the derivatives. BV stands for functions (which a.e. are equal to a function) of bounded variation.

TheOREM 1. Let (1.1)-(1.5) hold for some $p>0$. Then

and

$$
\lim _{t \rightarrow \infty} t^{p}\left(|x(t)|+\left|x^{\prime}(t)\right|\right)=0,
$$

$$
\int_{0}^{\infty} t^{2 p}\left(|x(t)|^{2}+\left|x^{\prime}(t)\right|^{2}+\left|x^{\prime \prime}(t)\right|^{2}\right) d t<\infty
$$

Received by the editors January 8, 1978.

AMS (MOS) subject classifications (1970). Primary 45D05, 45G99, 45J05, 45M05.

(C) 1980 American Mathematical Society $0002-9947 / 80 / 0000-0162 / \$ 03.00$ 
Theorem 1 is an extension of [10, Theorem 1] (which in turn extends [9, Theorem 4.3]). Essentially, putting $p=0$ in Theorem 1 we obtain [10, Theorem 1]. This work, as well as [10], has been inspired by MacCamy's [2] and [3], and Theorem 1 may be considered as a certain generalization of [2, Lemma 5.1] (when $\alpha=0$ ) and [3, Theorem II] (when $\alpha \neq 0$ ). The conclusions of [2, Lemma 5.1] and [3, Theorem II], rewritten for our scalar equation (1.1), are more or less equivalent to (1.6), (1.7) (with $p$ replaced by $m / 2$; MacCamy only discusses the case when $m$ is an integer). However, MacCamy's hypotheses are stronger than (1.1)-(1.5). Maybe the most substantial difference is that MacCamy replaces (1.3) by

$$
(1+t)^{2 p+3} a_{0}^{(k)}(t) \in L^{1}\left(R^{+}\right) \quad(k=0,1,2,3),
$$

(to correct a minor error in the proof of [2, Lemma 3.1] one has to strengthen [2, Condition (a $a_{2}$ ] to (1.8); cf. [1]). In addition, in [2] MacCamy strengthens (1.4) to

$$
(1+t)^{2 p} f(t),(1+t)^{2 p} f^{\prime}(t) \in L^{1} \cap L^{2} \cap L^{\infty}\left(R^{+}\right),
$$

and in [3] he requires more smoothness of $g$.

In the linear case $g(x)=k x$, where $k>0$, one can derive Theorem 1 from [6, Theorem 3] (and the hypotheses can be weakened).

MacCamy and Wong [4] treat (1.1) as a perturbation of a linear equation, and they give (global) conditions which imply $x(t)=O\left(e^{-\alpha t}\right)(t \rightarrow \infty)$ for some constant $\alpha>0$.

2. Some inequalities. Below we collect a number of inequalities which are needed in the proof of Theorem 1. In two of these inequalities the quadratic form

$$
Q(\varphi, T)=\int_{0}^{T} \varphi(t) \int_{0}^{t} a_{0}(t-s) \varphi(s) d s d t
$$

plays an important role. The versions given here are not the best possible (e.g., the requirement $\varphi \in C\left(R^{+}\right)$could be weakened).

LEMMA 2.1 [9, Proposition 4.1]. Let $a_{0}$ be strongly positive definite, and let $f, f^{\prime} \in L^{2}\left(R^{+}\right)$. Then there exists a constant $C$ such that for every $\varphi \in C\left(R^{+}\right)$and every $T \in R^{+}$,

$$
\left|\int_{0}^{T} \varphi(t) f(t) d t\right|^{2}<C Q(\varphi, T) .
$$

Our next inequality is a modification of [8, Lemma 1 and Theorem 2(ii)], and it has been used earlier in the proof of [9, Theorem 4.3] (although it is not explicitly formulated there). For completeness we outline a proof.

LEMMA 2.2. Let $a_{0}$ be strongly positive definite, and let $k \in L^{1} \cap \operatorname{BV}\left(R^{+}\right)$. Then there exists a constant $C$ such that for every $\varphi \in C\left(R^{+}\right)$and every $T \in R^{+}$,

$$
\int_{0}^{T}\left|\int_{0}^{t} k(t-s) \varphi(s) d s\right|^{2} d t<C Q(\varphi, T)
$$

OUTLINe OF PROOF. Define $\varphi_{T}(t)=\varphi(t)$ for $t \in[0, T], \varphi_{T}(t)=0$ otherwise, and put $\hat{\varphi}_{T}(\omega)=\int_{0}^{\infty} e^{-i \omega t} \varphi_{T}(t) d t, \hat{k}(\omega)=\int_{0}^{\infty} e^{-i \omega t} k(t) d t(\omega \in R)$. The strong positive definiteness of $a_{0}$ implies that for some $\varepsilon>0$, 


$$
Q(\varphi, T)>\varepsilon \int_{-\infty}^{\infty}\left(1+\omega^{2}\right)^{-1}\left|\hat{\varphi}_{T}(\omega)\right|^{2} d \omega
$$

(cf. [5, Corollary 2.1 and line (2.3)]). On the other hand, $k \in L^{1} \cap \mathrm{BV}\left(R^{+}\right)$implies that $|\hat{k}(\omega)|^{2}<C_{1}\left(1+\omega^{2}\right)^{-1}(\omega \in R)$ for some constant $C_{1}$. Use this, the fact that the Fourier transform maps convolution into pointwise multiplication, and the Plancherel identity to get

$$
\begin{aligned}
\int_{0}^{T}\left|\int_{0}^{t} k(t-s) \varphi(s) d s\right|^{2} d t & <\int_{0}^{\infty}\left|\int_{0}^{t} k(t-s) \varphi_{T}(s) d s\right|^{2} d t \\
& =\frac{1}{2 \pi} \int_{-\infty}^{\infty}|\hat{k}(\omega)|^{2}\left|\hat{\varphi}_{T}(\omega)\right|^{2} d \omega \\
& <\frac{C_{1}}{2 \pi} \int_{-\infty}^{\infty}\left(1+\omega^{2}\right)^{-1}\left|\hat{\varphi}_{T}(\omega)\right|^{2} d \omega
\end{aligned}
$$

Together, (2.3) and (2.4) imply (2.2) with $C=C_{1} /(2 \pi \varepsilon)$.

Finally, we shall need an estimate on the function

$$
k_{q-1}(t)=\int_{0}^{t} a_{0}(t-s)\left[(1+t)^{q / 2}-(1+s)^{q / 2}\right] \varphi(s) d s \quad\left(t \in R^{+}\right)
$$

This estimate is somewhat similar to one in [2, p. 11].

LEMMA 2.3. (i) Let $q>0$, and define $a_{q}(t)=(1+t)^{q / 2} a_{0}(t), \varphi_{q-2}(t)=(1+$ $t)^{(q-2) / 2} \varphi(t)\left(t \in R^{+}\right)$. Assume that $a_{q} \in L^{1}\left(R^{+}\right), \varphi_{q-2}, \varphi \in L^{2}\left(R^{+}\right)$. Then $k_{q-1} \in$ $L^{2}\left(R^{+}\right)$.

(ii) Let the hypothesis of (i) hold. In addition, suppose that $a_{q}^{\prime} \in L^{1}\left(R^{+}\right)$. Then $k_{q-1}^{\prime} \in L^{2}\left(R^{+}\right)$.

Observe that the conclusion of Lemma 2.3 holds trivially when $q=0$, as obviously $k_{-1} \equiv 0$. Also note that the assumption on $\varphi_{q-2}$ and $\varphi$ is equivalent to $\varphi_{q-2} \in L^{2}\left(R^{+}\right)$for $q>2$, and to $\varphi \in L^{2}\left(R^{+}\right)$for $q<2$. In particular, for $q>1$ it is implied by $\varphi_{q-1} \in L^{2}\left(R^{+}\right)$, where $\varphi_{q-1}(t)=(1+t)^{(q-1) / 2} \varphi(t)\left(t \in R^{+}\right)$.

In the proof of Lemma 2.3 we use the fact that $0<s<t$ and $r \in R$ implies

$$
(1+t)^{r-1}<C_{r}\left[(1+s)^{r-1}+(1+t-s)^{r-1}\right] \text {, }
$$

where $C_{r}=\max \left\{1,2^{r-2}\right\}$. This inequality is trivial when $r<1$. To prove it when $r>1$ one divides $(2.6)$ by $(2+t)^{r-1}$, substitutes $x=(1+s) /(2+t)$, and minimizes the function $x^{r-1}+(1-x)^{r-1}$ for $x \in[0,1]$.

Proof of Lemma 2.3. To simplify the notations, replace below the exponent $q / 2$ by $r$, i.e. define $r=q / 2$.

(i) First consider the case $0<r<1$, i.e. $0<q<2$. Then $0<s<t$ implies

$$
0<(1+t)^{r}-(1+s)^{r}<(1+t-s)^{r}
$$

(the first inequality is valid for $r>0$, and the second follows from (2.6), since $C_{r}=1$ for $0<r<1$ ). Thus

$$
\left|k_{q-1}(t)\right|<\int_{0}^{t}\left|a_{q}(t-s) \varphi(s)\right| d s
$$

and so, by Young's inequality, $k_{q-1} \in L^{2}\left(R^{+}\right)$. 
Next consider the case $r>1$, i.e. $q>2$. Clearly $0<s<t$ implies

$$
\begin{aligned}
0 & <(1+t)^{r}-(1+s)^{r}=r \int_{s}^{t}(1+v)^{r-1} d v \\
& \leqslant r(t-s)(1+t)^{r-1},
\end{aligned}
$$

which combined with (2.5), (2.6) gives

$$
\left|k_{q-1}(t)\right| \leqslant r C_{r}\left[\int_{0}^{t}\left|a_{2}(t-s) \varphi_{q-2}(s)\right| d s+\int_{0}^{t}\left|a_{q}(t-s) \varphi(s)\right| d s\right] .
$$

Thus, by Young's inequality, we again get $k_{q-1} \in L^{2}\left(R^{+}\right)$, and the proof of (i) is complete.

(ii) Differentiate $k_{q-1}$ to get $k_{q-1}^{\prime}=h_{1}+h_{2}$, where

$$
\begin{aligned}
& h_{1}(t)=\int_{0}^{t} a_{0}^{\prime}(t-s)\left[(1+t)^{r}-(1+s)^{r}\right] \varphi(s) d s, \\
& h_{2}(t)=r(1+t)^{r-1} \int_{0}^{t} a_{0}(t-s) \varphi(s) d s .
\end{aligned}
$$

By part (i) of Lemma 2.3, with $a_{0}$ replaced by $a_{0}^{\prime}, h_{1} \in L^{2}\left(R^{+}\right)$. The fact that $h_{2} \in L^{2}\left(R^{+}\right)$also follows directly from (2.6) and Young's inequality. This completes the proof of Lemma 2.3.

3. Proof of Theorem 1. We begin by introducing some notation. Define

$$
\varphi(t)=g(x(t)), \quad \psi(t)=\sqrt{\alpha} \int_{0}^{t} \varphi(s) d s \quad\left(t \in R^{+}\right)
$$

and put

$$
\gamma_{q}(t)=(1+t)^{q / 2} \gamma(t) \quad\left(t \in R^{+}\right),
$$

where $q>-1$, and $\gamma$ is one of the functions $x, x^{\prime}, x^{\prime \prime}, \varphi, \psi, a_{0}, f$ and $f^{\prime}$ (i.e., $x_{q}(t)=(1+t)^{q / 2} x(t),\left(x^{\prime}\right)_{q}(t)=(1+t)^{q / 2} x^{\prime}(t)$ etc.; in particular, $a_{q}(t)=$ $\left.(1+t)^{q / 2} a_{0}(t)\right)$. Moreover, for $q$ as above, define

$$
G_{q}(t)=(1+t)^{q} \int_{0}^{x(t)} g(\xi) d \xi \quad(t \in R)
$$

(observe that the exponent in the definition of $G_{q}$ is $q$, whereas in all the other cases it was $q / 2$ ).

We first give an induction proof of Theorem 1 in the case when $2 p$ is an integer (the case when $2 p$ is not an integer is treated in $\$ 4$ ). One initiates the induction by showing that

$$
x, x^{\prime}, \psi \in L^{2} \cap L^{\infty}\left(R^{+}\right)
$$

Our induction hypothesis is

$$
x_{q-1},\left(x^{\prime}\right)_{q-1}, \psi_{q-1} \in L^{2} \cap L^{\infty}\left(R^{+}\right),
$$

and it implies

$$
x_{q},\left(x^{\prime}\right)_{q}, \psi_{q} \in L^{2} \cap L^{\infty}\left(R^{+}\right),
$$

as long as $1<q<2 p$. By applying the induction step $2 p$ times one gets

$$
x_{2 p},\left(x^{\prime}\right)_{2 p} \in L^{2} \cap L^{\infty}\left(R^{+}\right) .
$$


At the end of this section we show that (3.4) implies (1.6), (1.7) (in this last step $2 p$ need not be an integer).

We omit the proof of the initial step, as it is easily obtained from the proof of the general step (i.e. take $q=0$, and observe that all terms with subindex -1 drop out from the estimates).

We are now ready to begin with the proof of the induction step. In addition to (1.1)-(1.5), assume that (3.2) holds for some $q, 1<q<2 p$. (For the moment we think of $q$ as an integer, but the same argument applies when $q$ is real.) We deduce from (1.5) and (3.2) that

$$
\varphi_{q-1} \in L^{2}\left(R^{+}\right), \quad G_{q-1} \in L^{1}\left(R^{+}\right) .
$$

Write (1.1) in the form (multiply by $(1+t)^{q / 2}$ )

$$
\left(x^{\prime}\right)_{q}+\sqrt{\alpha} \psi_{q}+w_{q}=f_{q}
$$

where

$$
w_{q}(t)=(1+t)^{q / 2} \int_{0}^{t} a_{0}(t-s) \varphi(s) d s \quad\left(t \in R^{+}\right) .
$$

Multiply (3.6) by $\varphi_{q}$ and integrate over $[0, T]$ to get

$$
\begin{aligned}
G_{q}(T)+\frac{1}{2}[ & \left.\psi_{q}(T)\right]^{2}+Q\left(\varphi_{q}, T\right) \\
= & G_{q}(0)+q \int_{0}^{T} G_{q-1}(t) d t+\frac{q}{2} \int_{0}^{T}\left[\psi_{q-1}(t)\right]^{2} d t \\
& +\int_{0}^{T} \varphi_{q}(t)\left[f_{q}(t)-k_{q-1}(t)\right] d t,
\end{aligned}
$$

where $Q$ and $k_{q-1}$ are defined as in (2.1) and (2.5). Hence, by (3.2) and (3.5),

$$
G_{q}(T)+\frac{1}{2}\left[\psi_{q}(T)\right]^{2}+Q\left(\varphi_{q}, T\right)<C+\int_{0}^{T} \varphi_{q}(t)\left[f_{q}(t)-k_{q-1}(t)\right] d t
$$

where $C$ is some constant independent of $T$.

To simplify the notation below we shall use the letter $C$ in the same way as in (3.8), namely, $C$ represents a constant independent of $T$. The actual value of $C$ may change from one line to the next.

Observe that all terms on the left-hand side of (3.8) are nonnegative. Using Lemma 2.1 together with (1.2)-(1.4), (3.5) and Lemma 2.3 one finds that

$$
G_{q}(T)+\frac{1}{2}\left[\psi_{q}(T)\right]^{2}+Q\left(\varphi_{q}, T\right)<C .
$$

In particular,

$$
G_{q}, \psi_{q} \in L^{\infty}\left(R^{+}\right)
$$

and

$$
Q\left(\varphi_{q}, T\right)<C
$$

Combining (1.5) with (3.9) we further deduce that

$$
x_{q}, \varphi_{q} \in L^{\infty}\left(R^{+}\right) \text {. }
$$


Moreover we claim that

$$
w_{q} \in L^{2} \cap L^{\infty}\left(R^{+}\right), \quad\left(x^{\prime}\right)_{q} \in L^{\infty}\left(R^{+}\right) .
$$

By (2.5) and (3.7)

$$
w_{q}(t)=\int_{0}^{t} a_{0}(t-s) \varphi_{q}(s) d s+k_{q-1}(t) \quad\left(t \in R^{+}\right) .
$$

That $\int_{0}^{t} a_{0}(t-s) \varphi_{q}(s) d s \in L^{2} \cap L^{\infty}\left(R^{+}\right)$follows from (1.2), (1.3), (3.10), (3.11) and Lemma 2.2. Also by (1.3), (3.5) and Lemma 2.3, $k_{q-1} \in L^{2} \cap L^{\infty}\left(R^{+}\right)$. Thus $w_{q} \in L^{2} \cap L^{\infty}\left(R^{+}\right)$. Together with (1.4) (which implies $f_{q} \in L^{\infty}\left(R^{+}\right)$), (3.6) and (3.9) this yields $\left(x^{\prime}\right)_{q} \in L^{\infty}\left(R^{+}\right)$and completes the proof of (3.12).

By now we have obtained the $L^{\infty}$-estimates in (3.3), but the crucial $L^{2}$-estimates are still missing. To get these we have to develop two more basic inequalities ((3.13) and (3.17) below). First, multiply (3.6) by $\left(x^{\prime}\right)_{q}$ and integrate over $[0, T]$ to get

$$
\begin{aligned}
\int_{0}^{T}\left|\left(x^{\prime}\right)_{q}(t)\right|^{2} d t-\alpha \int_{0}^{T} x_{q}(t) \varphi_{q}(t) d t \\
=-\sqrt{\alpha} x_{q}(T) \psi_{q}(T)+q \sqrt{\alpha} \int_{0}^{T} x_{q-1}(t) \psi_{q-1}(t) d t \\
+\int_{0}^{T}\left(x^{\prime}\right)_{q}(t)\left[f_{q}(t)-w_{q}(t)\right] d t
\end{aligned}
$$

Hence, by (1.4), (3.2), (3.9), (3.11), (3.12) and the Schwarz inequality,

$$
\int_{0}^{T}\left|\left(x^{\prime}\right)_{q}(t)\right|^{2}-\alpha \int_{0}^{T} x_{q}(t) \varphi_{q}(t) d t<C\left(1+\left[\int_{0}^{T}\left|\left(x^{\prime}\right)_{q}(t)\right|^{2} d t\right]^{1 / 2}\right) .
$$

Unfortunately, the second term on the left-hand side of (3.13) has the wrong sign (except when $\alpha=0$ ), and to take care of this term we have to produce one more inequality. Differentiate (1.1), and multiply it by $(1+t)^{q / 2}$ to get

$$
\left(x^{\prime \prime}\right)_{q}+\left(\alpha+a_{0}(0)\right) \varphi_{q}=\left(f^{\prime}\right)_{q}-v_{q},
$$

where

$$
v_{q}(t)=(1+t)^{q / 2} \int_{0}^{t} a_{0}^{\prime}(t-s) \varphi(s) d s \quad\left(t \in R^{+}\right) .
$$

We claim that

$$
v_{q} \in L^{2}\left(R^{+}\right) \text {. }
$$

Write $v_{q}$ in the form

$$
v_{q}(t)=\int_{0}^{t} a_{0}^{\prime}(t-s) \varphi_{q}(s) d s+\int_{0}^{t} a_{0}^{\prime}(t-s)\left[(1+t)^{q / 2}-(1+s)^{q / 2}\right] \varphi(s) d s .
$$

Using (1.2), (1.3), (3.5), (3.10), Lemma 2.2 applied to the first term and Lemma 2.3(i) (with $a_{0}$ replaced by $a_{0}^{\prime}$ ) applied to the second term in the right-hand side of (3.16) one gets (3.15).

Multiply (3.14) by $x_{q}$, and integrate over $[0, T]$ to get

$$
\begin{aligned}
\left(\alpha+a_{0}(0)\right) \int_{0}^{T} x_{q}(t) \varphi_{q}(t) d t-\int_{0}^{T}\left|\left(x^{\prime}\right)_{q}(t)\right|^{2} d t & \\
= & -x_{q}(T) x_{q}^{\prime}(T)+x(0) x^{\prime}(0)+q \int_{0}^{T} x_{q-1}(t)\left(x^{\prime}\right)_{q-1}(t) d t \\
& +\int_{0}^{T} x_{q}(t)\left[\left(f^{\prime}\right)_{q}(t)-v_{q}(t)\right] d t .
\end{aligned}
$$


Hence by (1.4), (3.2), (3.11), (3.12), (3.15) and the Schwarz inequality,

$$
\begin{aligned}
\left(\alpha+a_{0}(0)\right) \int_{0}^{T} x_{q}(t) \varphi_{q}(t) d t- & \int_{0}^{T}\left|\left(x^{\prime}\right)_{q}(t)\right|^{2} d t \\
& <C\left(1+\left[\int_{0}^{T}\left|x_{q}(t)\right|^{2} d t\right]^{1 / 2}\right)
\end{aligned}
$$

The strong positive definiteness of $a_{0}$ implies $a_{0}(0)>0$. Fix $\varepsilon>0$ so small that $a_{0}(0)>\varepsilon \alpha$, multiply (3.13) by $(1+\varepsilon)$, and add the result to (3.17). This gives

$$
\begin{aligned}
& \left(a_{0}(0)-\varepsilon \alpha\right) \int_{0}^{T} x_{q}(t) \varphi_{q}(t) d t+\varepsilon \int_{0}^{T}\left|\left(x^{\prime}\right)_{q}(t)\right|^{2} d t \\
& \quad<C\left(1+\left[\int_{0}^{T}\left|x_{q}(t)\right|^{2} d t\right]^{1 / 2}+\left[\int_{0}^{T}\left|\left(x^{\prime}\right)_{q}(t)\right|^{2} d t\right]^{1 / 2}\right)
\end{aligned}
$$

or equivalently (redefine $C$ ),

$$
\begin{aligned}
\int_{0}^{T} x_{q}(t) \varphi_{q}(t) & +\int_{0}^{T}\left|\left(x^{\prime}\right)_{q}(t)\right|^{2} d t \\
& <C\left(1+\left[\int_{0}^{T}\left|x_{q}(t)\right|^{2} d t\right]^{1 / 2}+\left[\int_{0}^{T}\left|\left(x^{\prime}\right)_{q}(t)\right|^{2} d t\right]^{1 / 2}\right)
\end{aligned}
$$

It follows from (1.5) and (3.11) that

$$
\left|x_{q}(t)\right|^{2}<C x_{q}(t) \varphi_{q}(t) \quad\left(t \in R^{+}\right)
$$

and so we finally have

$$
\begin{aligned}
\int_{0}^{T}\left|x_{q}(t)\right|^{2} d t & +\int_{0}^{T}\left|\left(x^{\prime}\right)_{q}(t)\right|^{2} d t \\
& <C\left(1+\left[\int_{0}^{T}\left|x_{q}(t)\right|^{2} d t\right]^{1 / 2}+\left[\int_{0}^{T}\left|\left(x^{\prime}\right)_{q}(t)\right|^{2} d t\right]^{1 / 2}\right)
\end{aligned}
$$

This implies

$$
x_{q},\left(x^{\prime}\right)_{q} \in L^{2}\left(R^{+}\right)
$$

Combining (1.4), (3.6), (3.9), (3.11), (3.12) and (3.18) one gets (3.3), and the proof of the induction step is complete.

We assert that (3.4) implies (1.6), (1.7) for arbitrary real values of $p>0$. As (1.6) is a consequence of (1.7), it suffices to show that (1.7) holds. But (3.14) (with $q=2 p$ ) combined with (1.4), (1.5), (3.4) and (3.15) (with $q=2 p$ ) imply $\left(x^{\prime}\right)_{2 p} \in$ $L^{2}\left(R^{+}\right)$(observe that (3.15) follows from (1.3), (2.6) (with $r-1=q / 2$ ), the fact that $\varphi_{q} \in L^{2}\left(R^{+}\right)$and Young's inequality). Hence (1.7) holds.

In particular, the proof of Theorem 1 in the case when $2 p$ is an integer is complete.

4. Nonintegral values of $2 p$. Examining the proof of the induction step given in $\$ 3$ one observes that it is also valid for nonintegral values of $q$, as long as $1<q<2 p$ (for $0<q<1$ one needs more than (3.5) in order to be able to apply Lemma 2.3).

To prove Theorem 1 when $2 p$ is real, $2 p>1$, one first applies Theorem 1 with $2 p$ replaced by the largest integer $m$ satisfying $m<2 p$. This yields (3.3) with $q$ replaced by $m$. However, as $2 p-1<m$, this implies (3.2) with $q=2 p$, and so one final application of the induction step gives (3.4), hence (1.6), (1.7). 
For $0<2 p<1$ the argument has to be modified slightly. That (3.1) holds is proved in the same way as before. In the proof of the general induction step, replace (3.2) and (3.5) by (3.1) and

$$
\varphi \in L^{2}\left(R^{+}\right), \quad G_{0} \in L^{1}\left(R^{+}\right),
$$

and take $q=2 p$. Then the argument remains valid, and we again obtain (3.4).

\section{REFERENCES}

1. C. M. Dafermos and J. A. Nohel, Energy methods for nonlinear hyperbolic Volterra integrodifferential equations, Comm. Partial Differential Equations 4 (1979), 219-278.

2. R. C. MacCamy, An integro-differential equation with applications in heat flow, Quart. Appl. Math. 35 (1977), 1-19.

3. 21-33.

, A model for one-dimensional, nonlinear viscoelasticity, Quart. Appl. Math. 35 (1977),

4. R. C. MacCamy and J. S. W. Wong, Exponential stability for a nonlinear functional differential equation, J. Math. Anal. Appl. 39 (1972), 699-705.

5. J. A. Nohel and D. F. Shea, Frequency domain methods for Volterra equations, Advances in Math. 22 (1976), 278-304.

6. D. F. Shea and S. Wainger, Variants of the Wiener-Lévy theorem, with applications to stability problems for some Volterra integral equations, Amer. J. Math. 97 (1975), 312-343.

7. O. J. Staffans, Positive definite measures with applications to a Volterra equation, Trans. Amer. Math. Soc. 218 (1976), 219-237.

8. __, An inequality for positive definite Volterra kernels, Proc. Amer. Math. Soc. 58 (1976), 205-210.

9. __ Boundedness and asymptotic behavior of solutions of a Volterra equation, Michigan Math. J. 24 (1977), 77-95.

10. __ A nonlinear Volterra integral equation with square integrable solutions, Volterra Equations, Lecture Notes in Math., vol. 737, Springer-Verlag, Berlin and New York, 1979, pp. 281-286.

Department of Mathematics, Helsink University of Technology, SF-02150 Espoo 15, Finland 